\title{
Morfologia das papilas linguais de coelhos Oryctolagus cuniculus (Lagomorpha: Leporidae)
}

\author{
Lorena Tannús Menezes ${ }^{1 *}$ \\ Árthur Paulino Sanzo Kaminishi ${ }^{1}$ \\ Lucélia Gonçalves Vieira ${ }^{1}$ \\ Sérgio Rodrigo Pereira de Oliveira ${ }^{1}$ \\ Caio Henrique Ferreira ${ }^{1}$ \\ Marcelo Emílio Beletti ${ }^{2}$ \\ André Luiz Quagliatto Santos ${ }^{1}$ \\ Universidade Federal de Uberlândia \\ Avenida Piauí, s/n, Bloco 4S, Jardim Umuarama, CEP 38400-902, Uberlândia - MG, Brasil \\ ${ }^{1}$ Laboratório de Ensino e Pesquisa em Animais Silvestres \\ ${ }^{2}$ Instituto de Ciências Biomédicas, Uberlândia \\ * Autor para correspondência \\ lorena_tannus@hotmail.com
}

Submetido em 01/10/2012

Aceito para publicação em 17/01/2013

\section{Resumo}

A língua representa a diversificação morfológica nos mamíferos; suas papilas possuem funções mecânicas e gustativas na alimentação. Este estudo objetivou descrever a morfologia macroscópica e microscópica das papilas linguais dos coelhos da espécie Oryctolagus cuniculus. Foram utilizadas 45 línguas dissecadas de coelhos adultos pertencentes ao acervo do Laboratório de Ensino e Pesquisa em Animais Silvestres (LAPAS) da Universidade Federal de Uberlândia (UFU). Para a identificação microscópica da língua foram utilizadas as técnicas de microscopia óptica e microscopia eletrônica de varredura (MEV). A língua possui quatro tipos de papilas: filiforme, fungiforme, valada e folhada. As papilas filiformes possuem função mecânica e apresentam três subtipos: tipo 1, com forma cônica, do corpo da língua até a margem rostral da proeminência lingual; tipo 2, com formato pontiagudo, na área rostral da proeminência lingual; e tipo 3, com forma larga e pontiaguda, na área caudal da proeminência lingual. As papilas fungiformes possuem função gustativa e estão no corpo da língua. As papilas folhadas são pares, possuem função gustativa, nas superfícies laterais do corpo da língua. As papilas valadas são pares, possuem função gustativa, na raiz. Na língua foi observado um epitélio estratificado pavimentoso queratinizado, que reveste as papilas e as regiões entre elas.

Palavras-chave: Anatomia; Língua; Microscopia de luz; Microscopia eletrônica de varredura

\section{Abstract}

Morphology of the lingual papillae of Oryctolagus cuniculus (Lagomorpha: Leporidae) rabbits. The tongue represents the morphological diversification in mammals; their papillae have mechanical and gustatory functions in feeding. This study aimed to describe the macroscopic and microscopic morphology of 
the lingual papillae of rabbits of the Oryctolagus cuniculus species. One used 45 dissected tongues from adult rabbits belonging to the collection of the Laboratory for Education and Research on Wild Animals (LAPAS) of Universidade Federal de Uberlandia (UFU). For the microscopic identification of the tongue, the optical microscopy and the scanning electron microscopy (SEM) techniques were used. The tongue has four papillae types: filiform, fungiform, vallate, and foliate. The filiform papillae have a mechanical function and they present three subtypes: type 1, with a conical shape, from the tongue's body to the rostral edge of the lingual prominence; type 2, with a sharp shape, in the rostral area of the lingual prominence; and type 3 , with a thick and sharp shape, in the caudal area of the lingual prominence. The fungiform papillae have a gustatory function and they're in the tongue's body. The foliate papillae are pairs, they have a gustatory function, in the lateral surfaces of the tongue's body. The vallate papillae are pairs, they have a gustatory function, in the root. On the tongue, one observed a keratinized squamous stratified epithelium, lining the papillae and the regions between them.

Key words: Anatomy; Light microscopy; Scanning electron microscopy; Tongue

\section{Introdução}

Os coelhos domésticos da raça Nova Zelândia Oryctolagus cuniculus são mamíferos, herbívoros não-ruminantes (ARRUDA et al., 2005), da ordem Lagomorpha e da família Leporidae, originados dos coelhos selvagens da região oeste da Europa e noroeste da África. Antes de ser classificado como Lagomorfa, o coelho pertencia ao grupo dos roedores (HARKNERS; WAGNER, 1993). Constitui uma importante fonte de proteína animal para a alimentação humana nos Estados Unidos e Europa e é considerado um animal convencional de laboratório, contribuindo para diversos estudos científicos (HERRERA et al., 2001).

As estruturas morfológicas das línguas dos vertebrados possuem diferentes funções, como a captura e manipulação de alimentos, higiene e vocalização (KILINC et al., 2010) sendo importantes para a adaptação das espécies (SONNTAG, 1920). Nos tetrápodes, a língua desempenha um papel importante na alimentação (IWASAKI, 2002) e apresenta grande diversificação morfológica nos mamíferos quanto ao seu tamanho e à sua forma (KEASTER, 1940), devido aos diferentes hábitos alimentares (SONNTAG, 1920; SISSON, 1986; DYCE et al., 1997) e isto contribui para a morfologia, distribuição e tipo das papilas (PASTOR et al., 2008).

Estudos da superfície da língua dos mamíferos mostraram a presença de várias papilas com distribuição peculiar (BENETTI et al., 2009). Papilas altamente diferenciadas, com funções mecânicas e gustativas (KONIG; LIEBICH, 2004). Nos mamíferos domésticos, as papilas linguais são distintas em sua morfologia como filiformes, fungiformes, valadas e folhadas. Os animais herbívoros têm filiformes, fungiformes e valadas, mas não possuem as papilas folhadas (ZHENG; KOBAYASHI, 2006). As papilas filiformes, cônicas e lentiformes têm funções mecânicas, enquanto as fungiformes, valadas e folhadas têm função gustativa (KONIG; LIEBICH, 2004).

Em um aspecto geral, a morfologia das papilas linguais já foi estudada nos coelhos (SILVA et al., 2002; KULAWIK; GODYNICKI, 2007a; 2007b; NONAKA et al., 2008), roedores (IINO; KOBAYASHI, 1988; SHINDO et al., 2006; KILINC et al., 2010), ruminantes (EERDUNCHAOLU et al., 2001; ZHENG; KOBAYASHI, 2006; GUIMARÃES et al., 2007; MAHABADY et al., 2010), carnívoros (EMURA et al., 2004; 2006; JACKOWIAK; GODYNICKI, 2004; JACKOWIAK et al., 2009) e primatas (KOBAYASHI et al., 2004; BURITY et al., 2009; BRANCO et al., 2011; 2012), no entanto, um estudo pormenorizado destas estruturas em coelhos ainda não foi realizado.

O objetivo neste estudo foi descrever a morfologia macroscópica e microscópica das papilas linguais dos coelhos da raça Nova Zelândia Oryctolagus cuniculus.

\section{Material e Métodos}

Foram utilizadas 45 línguas de coelhos domésticos Oryctolagus cuniculus (Linnaeus, 1758) adultos fixadas em solução aquosa de formaldeído a 3,7\%, as quais pertencem ao acervo didático-científico do Laboratório de Ensino e Pesquisa em Animais Silvestres (LAPAS) da Universidade Federal de Uberlândia (UFU). 
Em cada espécime, foram identificados o tipo, a forma e a distribuição das papilas linguais dos coelhos. Essas observações foram efetuadas com o auxílio de microscópio estereoscópico Coleman ${ }^{\circledR}$ (SQF-E).

Para a identificação macroscópica das papilas linguais, foi utilizada a impregnação com Lugol 3\% e fotografada para documentação com a câmera Sony (DSC-H10), e foi realizado um desenho esquemático para melhor elucidar a localização das estruturas estudadas.

Para a identificação das características microscópicas da língua, foi utilizada a técnica de microscopia óptica. Diante do exposto, foram coletados fragmentos do ápice, corpo, raiz, e das papilas linguais identificadas. Esses fragmentos foram mantidos em solução de formaldeído a $3,7 \%$, e posteriormente incluídos em parafina, seguindo a rotina histológica usual.

Os blocos obtidos foram cortados em micrótomo automático Leica (RM2155). Os cortes, corados em Hematoxilina/Eosina foram montados e analisados histologicamente. As fotomicrografias para a documentação foram obtidas em microscópio óptico com câmera interna Leica (DM500).

Para a identificação das papilas linguais, também foi utilizada a técnica de microscopia eletrônica de varredura (MEV). As amostras foram fixadas em uma solução aquosa de glutaraldeído a $2 \%$ e paraformaldeído a $2 \%$. Após a fixação, o material foi preservado em uma solução tampão de cacodilato de sódio a $0,1 \mathrm{M}$, aguardando o processo de pós-fixação, onde o material foi submerso em uma solução de tetróxido de ósmio a $1 \%$. Em seguida, o material passou por um processo de desidratação gradativa com banhos de soluções aquosas de acetona de 30, 50, 70 e $90 \%$ e três banhos de acetona pura, de 15 minutos cada, antes de sofrer a secagem ao ponto crítico com $\mathrm{CO}_{2}$ e metalização de sua superfície em um sputter coater. O material foi fotografado no microscópio Jeol (JSM-70001F).

\section{Resultados}

As línguas dos coelhos apresentaram uma forma alongada, com um ápice arredondado, com uma média de
6,0 cm de comprimento. Possuem três regiões distintas, uma mais caudal, a raiz que contém as papilas valadas; o corpo, região entre a raiz e o ápice que contém uma proeminência lingual, localizada na região intermolar; e uma região mais rostral, o ápice que é delimitado pelo frênulo lingual. O terço rostral da língua é demarcado por um sulco mediano, até a margem rostral da proeminência lingual (Figura 1).

Histologicamente foram detectados na língua uma túnica mucosa, glândulas serosas e mucosas, músculos, vasos e nervos (Figura 2A). Na raiz, corpo e ápice das línguas de coelhos foram possíveis observar um epitélio do tipo estratificado pavimentoso queratinizado que revestiu tanto as papilas como as regiões entre elas. Ainda, sob análises com microscopia de luz, foi possível confirmar a presença das papilas filiformes, fungiformes, folhadas e valadas na constituição da túnica mucosa dos coelhos (Figuras 2, 4C, 4D e 4E).

As papilas filiformes são projeções finas, semelhantes a filamentos. Não estão presentes na região da raiz da língua. Foram observados três diferentes subtipos: papilas filiformes do tipo 1, tipo 2 e tipo 3 (Figuras 2 e 3).

As papilas filiformes do tipo 1, estão distribuídas na superfície da língua até a margem rostral do torus lingual, intercaladas com as papilas fungiformes. Apresentam um formato cônico, com extremidade leve ou ausente. (Figuras 2B, 3A e 3B).

As papilas filiformes do tipo 2 estão presentes na proeminência lingual, onde não há presença de papilas fungiformes. Essas papilas estão distribuídas, especificamente, na margem rostral da proeminência lingual, são mais espessas que as papilas filiformes do tipo 1, ou seja, apresentam epitélio com maior quantidade de queratina, além de possuírem extremidades pontiagudas (Figuras 2C e 3C).

As papilas filiformes do tipo 3, também estão localizadas na proeminência lingual, no entanto, distribuem-se na área caudal da proeminência lingual. Possuem a base larga e uma extremidade fina e pontiaguda (Figura 3D). Devido a sua estrutura morfológica, pode ser considerada papila filiforme intermediária ao tipo 1 e ao tipo 2 (Figura 2D). 
FIGURA 1: Fotomacrografia da vista dorsal da língua do coelho. Em (A), evidenciando a visão geral e as regiões da língua (ápice, corpo e raiz). Em (B) enfatizando as papilas valadas, (C) folhadas, (D) filiformes presentes na proeminência lingual, (E) filiformes e fungiformes. Em (F) um desenho esquemático da localização das papilas linguais (vista dorsal). No corpo da língua existem as papilas filiformes do tipo 1 e o sulco mediano, na área rostral da proeminência lingual existem as papilas filiformes do tipo 2, e na parte caudal as papilas filiformes do tipo 3. Abreviaturas: va, valadas; fo, folhada, fi, filiformes, fu, fungiformes. Magnitude: A: 10X, BC: 30X, DE: 50X.

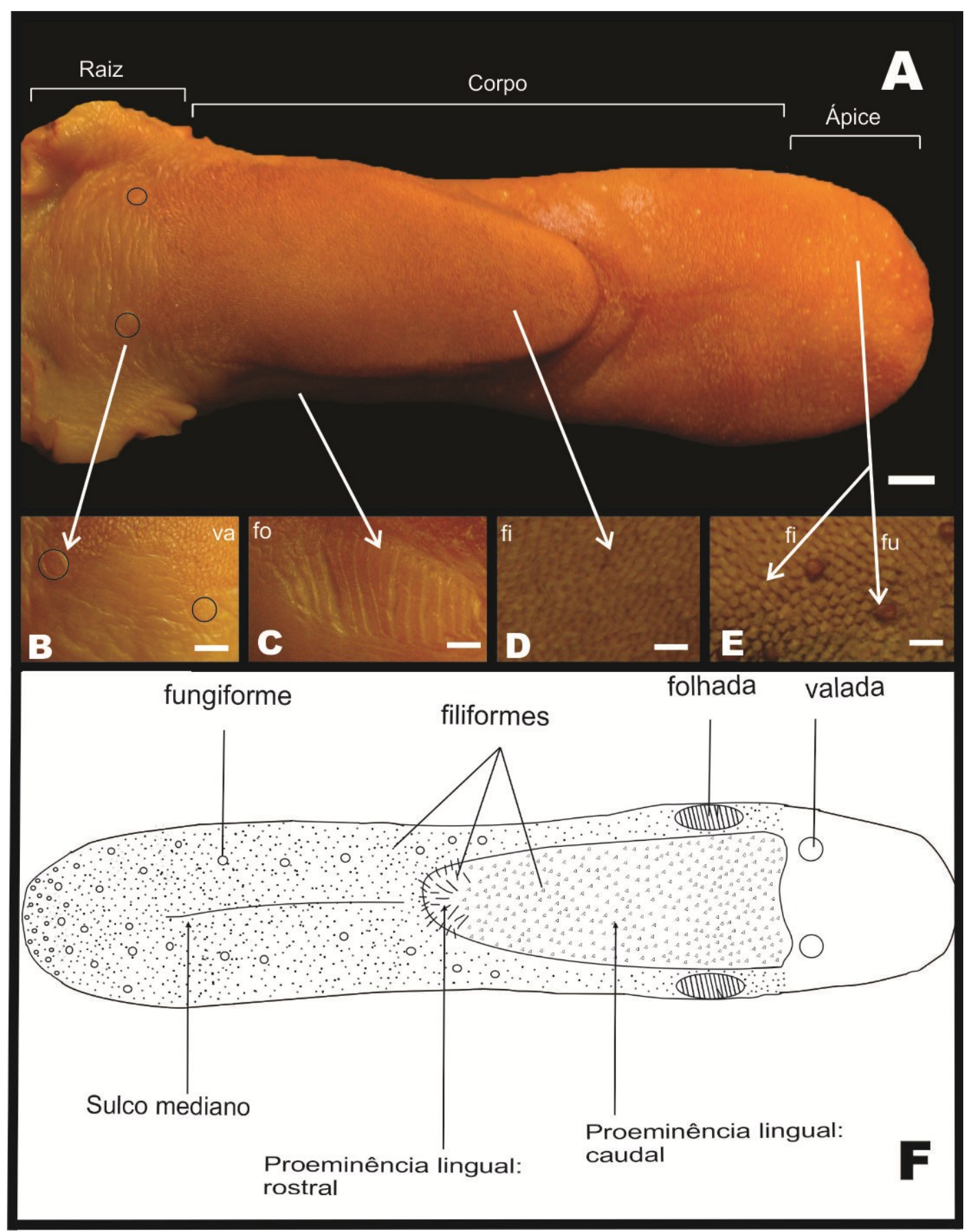


FIGURA 2: A: Fotomicrografia de um corte sagital da língua de coelhos; B: segmento do corpo da língua com epitélio tipo estratificado pavimentoso queratinizado das papilas filiformes do tipo 1 no corpo da língua; C: segmento rostral da proeminência lingual com epitélio tipo estratificado pavimentoso queratinizado das papilas filiformes do tipo 2; D: segmento rostral da proeminência lingual com epitélio tipo estratificado pavimentoso queratinizado das papilas filiformes do tipo 3. Abreviações: T: túnica mucosa; Mu: músculos; S: glândulas serosas; M glândulas mucosas. Coloração H.E.; Magnitude: A: 4X, Barra 500 m. BCD: 10X, Barra $200 \mu \mathrm{m}$.

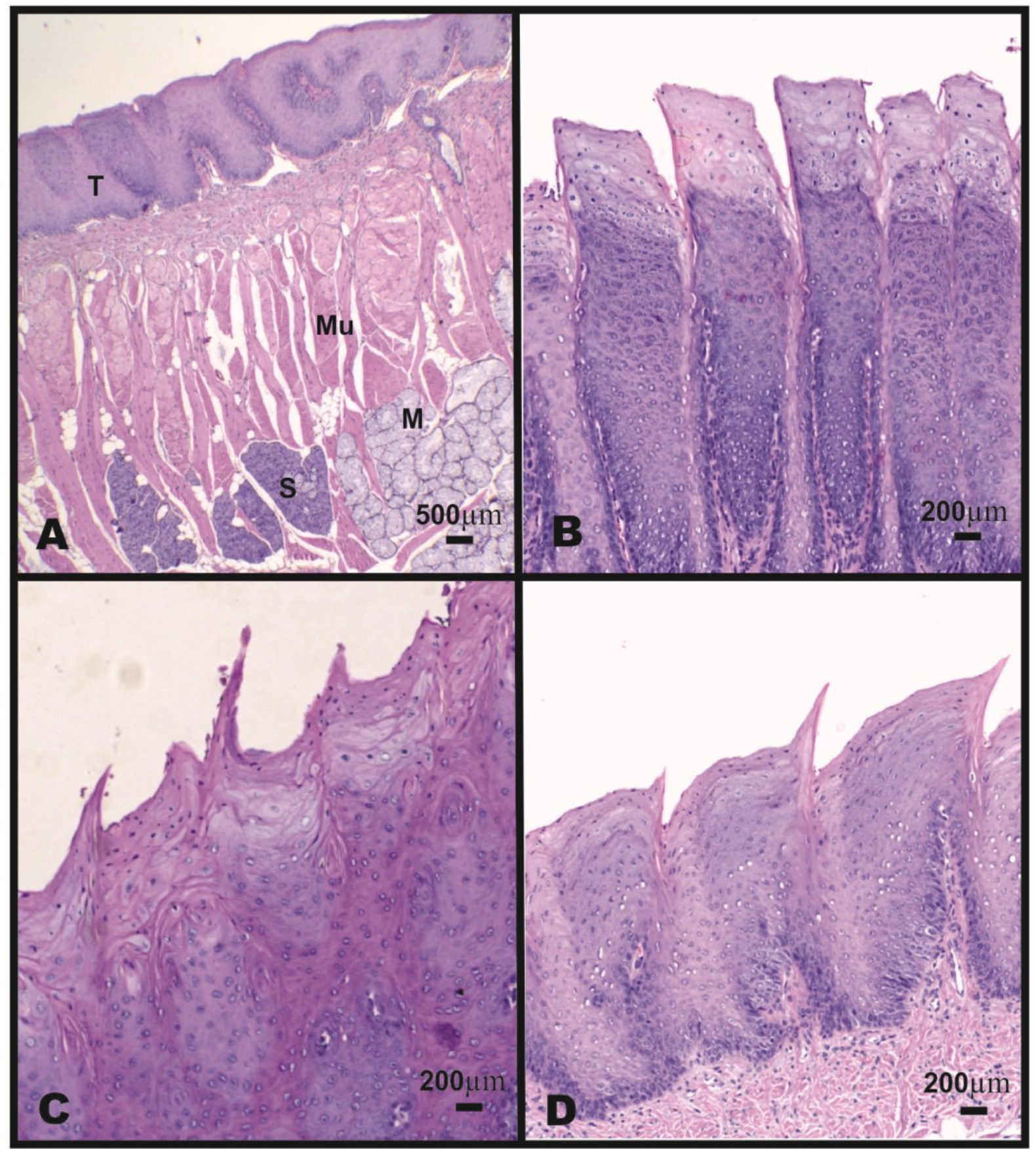


FIGURA 3: Fotomicrografia da forma externa do corpo da língua. A: papilas filiformes do tipo 1 intercaladas com as papilas fungiformes (setas) distribuídas no corpo da língua; B: papilas filiformes do tipo 1; C: papilas filiformes do tipo 2 distribuídas na área rostral da proeminência lingual; D: papilas filiformes do tipo 3 distribuídas na parte caudal da proeminência lingual; E: papila fungiforme distribuídas no corpo da língua. Barra: $100 \mu \mathrm{m}$, A: 55X; BDE: 400X; C: 120X.

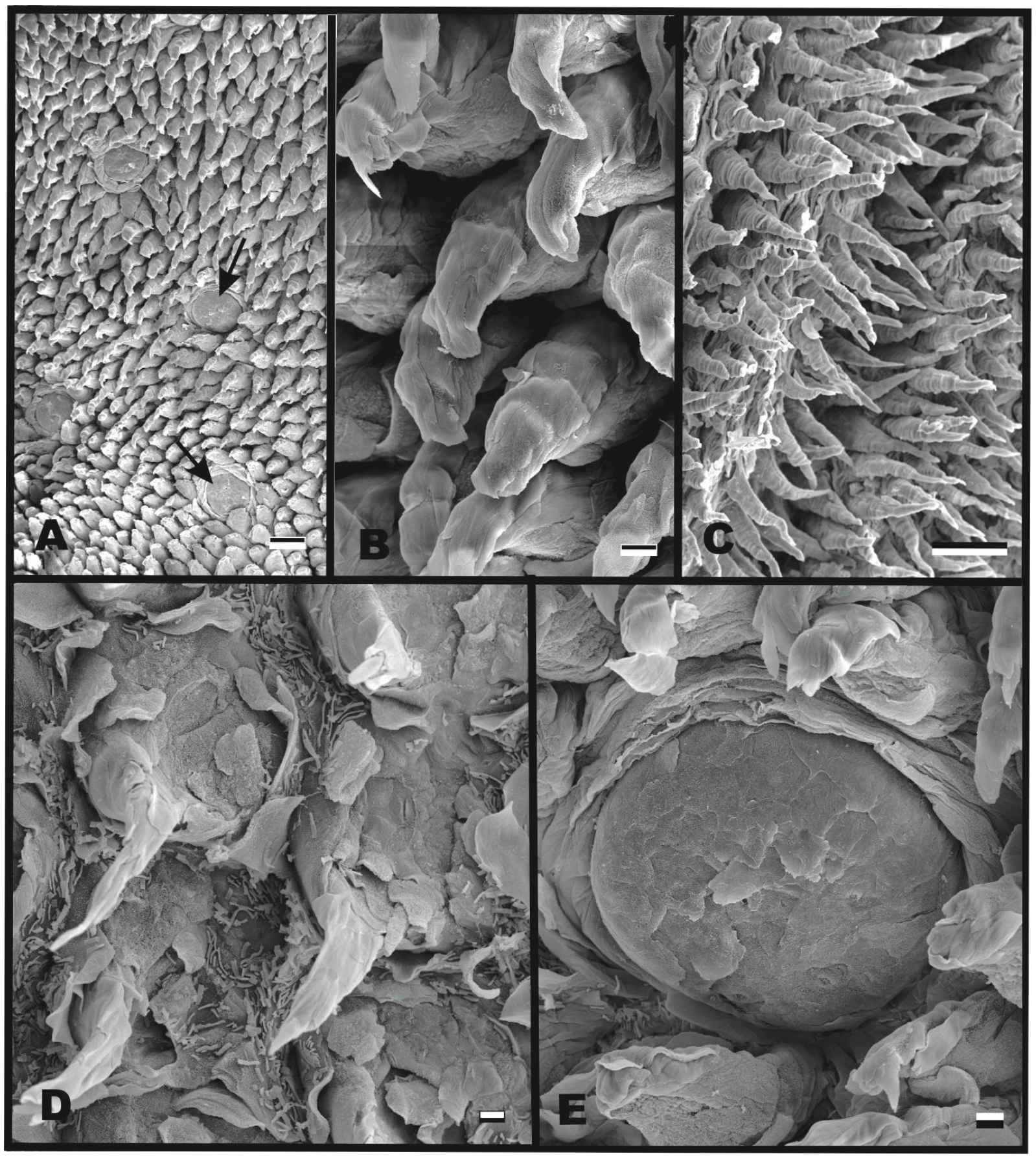


As papilas fungiformes são facilmente observadas, seu diâmetro é de $0,55 \mathrm{~mm}$ (Figura $3 \mathrm{E}$ ), são arredondadas na extremidade livre e sustentadas por um colo. Estão distribuídas aleatoriamente entre as papilas filiformes no ápice e no corpo da língua, são maiores que as papilas filiformes e se encontram em alta concentração na região do ápice e são ausentes na proeminência lingual. Possuem botões gustativos (Figura 4D).

FIGURA 4: A: Fotomicrografia da forma externa da papila folhada distribuída na superfície lateral da parte média da língua. São observados vários sulcos profundos e cristas paralelas; B: Fotomicrografia da forma externa da papila valada distribuída na raiz da língua. Barra: $100 \mu \mathrm{m}, \mathrm{A}: 55 \mathrm{X}$; B: 160X. C: Fotomicrografia de um corte sagital da superfície lateral das papilas folhadas distribuídas nas superfícies laterais da parte média da língua. São observados vários botões gustativos no epitélio lateral das papilas (seta); D: Fotomicrografia de um corte sagital de um segmento do corpo da língua das papilas fungiformes. São observados botões gustativos nas células epiteliais das papilas (seta); E: Fotomicrografia de um corte sagital da superfície da raiz da língua das papilas valadas. São observados vários botões gustativos no epitélio das papilas (seta). Coloração H.E., CDE: 10X, Barra $200 \mu \mathrm{m}$.

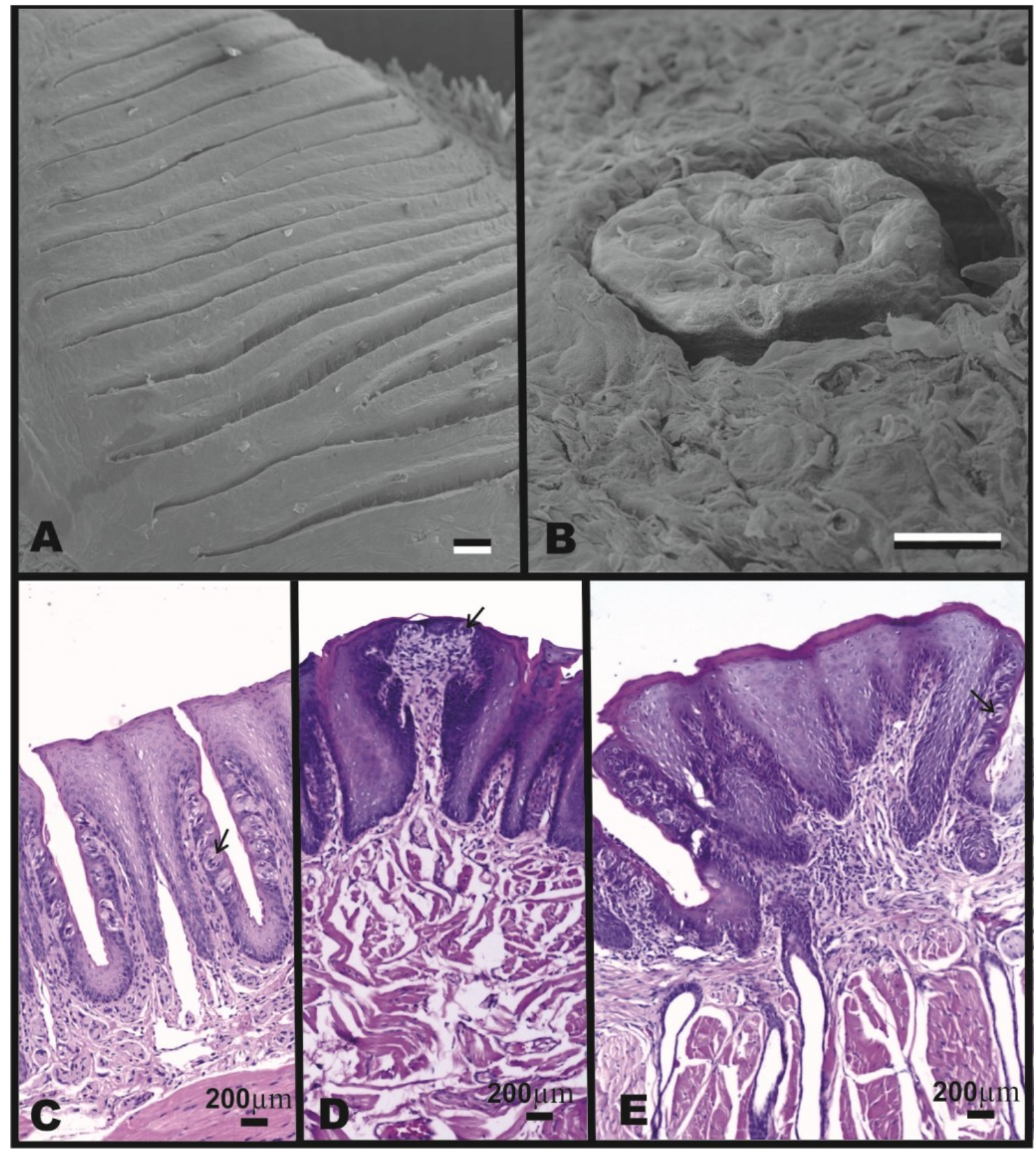


As papilas folhadas constituem um par, com formato elipsoide, com uma média de 15 sulcos profundos e cristas paralelas em forma de I (Figura 4A). Localizadas na região caudo-lateral do corpo da língua, se iniciam a partir do último dente molar. Possuem botões gustativos no epitélio lateral (Figura 4C).

As papilas valadas constituem um par, com $1,05 \mathrm{~mm}$ de diâmetro, são mais arredondadas e largas em sua superfície livre do que em sua porção inserida em forma de $\mathrm{V}$, estão mergulhadas numa depressão limitada por uma parede anular. Localizam-se na raiz da língua, caudalmente a proeminência lingual, entre os ramos da mandíbula. Possuem botões gustativos (Figuras 4B e 4E).

\section{Discussão}

As papilas filiformes e as fungiformes estão localizadas no ápice e no corpo da língua; as papilas folhadas na região caudo-lateral do corpo da língua e as papilas valadas em sua raiz. A presença e distribuição das papilas na superfície da língua dos coelhos é semelhante a dos búfalos (MAHADADY et al., 2010), dos coelhos (SILVA et al., 2002; NONAKA et al., 2008) e dos roedores (KILINIC et al., 2010; NASR et al., 2012).

A existência da proeminência lingual, na região intermolar no corpo da língua, como encontrado nos coelhos, é comum aos animais herbívoros, como outras espécies de coelhos (SILVA et al., 2002; NONAKA et al., 2008), roedores (SHINDO et al., 2006; NASR et al., 2012) e ruminantes (ABD-ELNAEIM et al., 2002; MAHADADY et al., 2010; EMURA et al., 2011a). Essa estrutura ajuda os animais herbívoros na mastigação dos alimentos (DYCE et al., 1997).

A proeminência lingual pode ser dividida em duas áreas de distribuição de papilas. $\mathrm{Na}$ área rostral existem as papilas filiformes do tipo 2, que são espessas, pontiagudas e com orientação rostral; e na área caudal da proeminência lingual, mais larga, existem as papilas filiformes do tipo 3, que possuem as bases largas e as extremidades pontiagudas. As papilas presentes na proeminência lingual são mais queratinizadas do que as papilas filiformes do tipo 1, aumentando o atrito do alimento com a superfície da língua durante a mastigação, possuem portanto, função mecânica na mastigação dos coelhos assim como em outros herbívoros (NICKEL et al., 1979; SISSON, 1986; DYCE et al., 1997; IWASAKI, 2002).

A morfologia e a estrutura das papilas filiformes das espécies evoluem de acordo com os requisitos e funções de mastigação (IWASAKI, 2002; NONAKA et al., 2008), a idade e hábitos alimentares (AGUNGPRIYONO et al., 1995). Neste estudo, observaram-se três subtipos de papilas filiformes: tipo 1, tipo 2 e tipo 3, que corroboram com Silva et al. (2002), porém, para Nonaka et al. (2008), apresentaram a mesma localização, e não a mesma estrutura. As papilas filiformes do tipo 1 dos coelhos estão densamente distribuídas em toda a superfície da língua até a margem rostral da proeminência lingual, exceto na região da raiz da língua. Uma disposição semelhante pode ser observada nos roedores (IINO; KOBAYASHI, 1988; KIM et al., 2003). Já os ruminantes (DE PAZ CABELLO et al., 1988; KURTUL; ATALGIN, 2008; MAHABADY et al., 2010), felídeos (OJIMA et al., 2000; EMURA et al., 2004) e os primatas (KOBAYASHI et al., 2004; BRANCO et al., 2011; 2012) apresentam distribuição diferenciada das papilas em relação aos coelhos.

As papilas filiformes do tipo 2 são semelhantes às papilas dos carnívoros (OJIMA et al., 2000; OJIMA, 2001; EMURA et al., 2004; JACKOWIAK; GODYNICKI, 2004). As papilas filiformes do tipo 3 se assemelham às dos ruminantes (TADJALLI; PAZHOOMAND, 2004; ZHENG; KOBAYASHI, 2006; KURTUL; ATALGIN, 2008).

As papilas fungiformes estão distribuídas aleatoriamente no ápice e no corpo da língua, e não estão presentes na proeminência lingual e na raiz da língua dos coelhos. Estão em alta concentração e tamanho no ápice, assim como nas espécies marten japonês (Martes melampus) (EMURA et al., 2007), cão-guaxinim (Nyctereutes procyonoides) (EMURA et al., 2006), tigre siberiano (Panthera tigris altaica) (EMURA et al., 2004), raposa vermelha (Vulpes vulpes fulva) (JACKOWIAK; GODYNICK, 2004) e raposa do ártico Alopex lagopus (JACKOWIAK et al., 2009). Nos porcos-da-índia Cavia porcellus (KOBAYASHI, 1990), 
preguiça de coleira Bradypus torquatus (BENETTI et al., 2009) e a lebre-patagônica Dolichotis patagonum (EMURA et al., 2011b) estão presentes apenas no ápice e nas superfícies laterais da língua.

Foram observados botões gustativos nas papilas fungiformes dos coelhos, isso confirma sua função gustativa na espécie, o mesmo foi observado em ratos (IINO; KOBAYASHI, 1988), cachorros (OJIMA, 2001) e primatas (KOBAYASHI et al., 2004), ao contrário dos achados de Souza et al. (2012) em quatis.

As papilas folhadas possuem sulcos profundos e cristas paralelas em formato de folhas com vários botões gustativos e estão localizadas na parte caudal das superfícies laterais da parte média da língua dos coelhos. Nos primatas (KOBAYASHI et al., 2004; BURITY et al., 2009), as papilas folhadas são muito desenvolvidas e com muitos botões gustativos. Conforme o encontrado no presente estudo, animais herbívoros como coelhos e roedores (STANGL; PFAU, 1994; SILVA et al., 2002; SHINDO et al., 2006) também apresentam papilas folhadas com muitos botões gustativos, confirmando sua função gustativa. Contradizendo o que Zheng e Kobayashi (2006) dizem que os animais herbívoros não possuem a papilas folhadas. Já os cães e os porcos (OJIMA, 2001; KUMAR; BATE, 2004) apresentam saliências lineares sem função paladar nenhuma, pois não apresentam botões gustativos.

As papilas valadas estão presentes na raiz da língua e o seu número se difere nas espécies animais. Alguns roedores possuem apenas uma papila valada (STANGL; PFAU, 1994; AGUNGPRIYONO et al., 1995; KILINC et al., 2010). Os coelhos, preás e porcos possuem duas papilas valadas (KOBAYASHI, 1990; KUMAR; BATE, 2004; KULAWIK; GODYNICKI, 2007b; NONAKA et al., 2008). Os coalas (KOBAYASHI et al., 2003), gambá-comum (MANÇANARES et al., 2012) e castor norte americano (SHINDO et al., 2006) possuem três papilas valadas; os marten japonês (EMURA et al., 2007) e os tigres siberianos (EMURA et al., 2004) possuem quatro papilas valadas; os quatis possuem nove (SOUZA et al., 2012) e os cães e gatos (SISSON, 1986; OJIMA, 2001) e os ruminantes (KUMAR et al., 1998; EERDUNCHAOLU, 2001; TADJALLI; PAZHOOMAND, 2004; ZHENG; KOBAYASHI, 2006;
KURTUL; ATALGIN, 2008) possuem várias papilas valadas. Quanto maior a quantidade de papilas valadas maior a sensibilidade ao paladar (KEASTER, 1940). Diferente do golfinho-do-rio-da-prata (GUIMARÃES et al., 2012) as papilas valadas dos coelhos possuem botões gustativos.

A distribuição das duas papilas valadas nos coelhos é uma de cada lado na região da raiz da língua, simétricas à linha dos ramos da mandíbula, essa disposição é semelhante aos preás Cavia porcellus (KOBAYASHI, 1990; KULAWIK; GODYNICKI, 2007b).

A partir dos resultados, foi possível concluir que a língua dos coelhos Oryctolagus cuniculus tem forma alongada, com um ápice arredondado, formada por três regiões distintas, o ápice, o corpo e a raiz. Foram observados quatro tipos de papilas: filiformes, fungiformes, folhadas e valadas, com distribuição padrão comum aos mamíferos.

\section{Agradecimentos}

À CAPES pela concessão da bolsa de Mestrado; ao Técnico Fabrício Faria Araújo da Histologia ICBIMUFU, pela ajuda na execução do processo histológico; à Técnica Ingrid Gracielle Martins da Silva do IBD-CEL da UnB, pela ajuda nas microfotografias em MEV; e a Ms. Priscilla Rosa Queiroz Ribeiro pela leitura e sugestões feitas no trabalho.

\section{Referências}

ABD-ELNAEIM, M. M. M.; ZAYED, A. E.; LEISER, R. Morphological characteristics of the tongue and its papillae in the donkey (Equus asinus): a light and scanning electron microscopical study. Annals of Anatomy - Anatomischer Anzeiger, Jena, v. 184, p. 473-480, 2002.

AGUNGPRIYONO, S.; YAMADA, J.; KITAMURA, N.; NISA, C.; SIGIT, K.; YAMAMOTO, Y. Morphology of the dorsal lingual in the lesser mouse deer Tragulus javaicus. Journal of Anatomy, Londres, v. 187, p. 635-640, 1995.

ARRUDA, A. M. V.; PEREIRA, E. S.; MIZUBUTI, I. Y.; LOPES, D. C.; SILVA, J. F. Digestibilidade de nutrientes em coelhos alimentados com rami (Bohemeria nivea). Semina: Ciências Agrárias, Londrina, v. 26, p. 581-590, 2005.

BENETTI, E. J.; PICOLI, L. C.; GUIMARAES, J. P.; MOTOYAMA, A. A.; MIGLINO, M. A.; WATANABE, L. S. Characteristics of filiform, fungiform and vallate papillae and surface of interface 
epithelium-connective tissue of the maned sloth tongue mucosa (Bradypus torquatus, Iliger, 1811): light and scanning electron microscopy study. Anatomia, Histologia, Embryologia, Berlin, v. 38, p. 42-48, 2009.

BRANCO, E.; GUIMARÃES, J. P.; MIGLINO, M. A.; LACRETA JR., A. C. C.; ISHIZAKI, M. N.; GOMES, B. D.; MUNIZ, J. A. P. C.; IMBELONI, A.; FIORETTO, E. T.; DE LIMA, A. R. Ultrastructural aspects of lingual papillae em squirrel monkey (Saimiri sciureus). Microscopy Research and Technique, New York, v. 74, p. 484-487, 2011.

BRANCO, E.; GUIMARÃES, J. P.; MIGLINO, M. A.; LACRETA JR., A. C. C.; ISHIZAKI, M. N.; GOMES, B. D.; MUNIZ, J. A. P. C.; IMBELONI, A.; FIORETTO, E. T.; DE LIMA, A. R. Ultrastructural aspects of Callithrix penicillata lingual papillae. Microscopy Research and Technique, New York, v. 75, n. 3, p. 282-284, 2012.

BURITY, C. H. F.; SILVA, M. R.; SOUZA, A. M.; LANCETTA, C. F. F.; MEDEIROS, M. F.; PISSINATTI, A. Scanning a electron microscopic study of the tongue in golden-headed tamaris, Leontopithecus chrysomelas (Callithrichidae: Primates). Zoologia, Curitiba, v. 26, p. 323-327, 2009.

DE PAZ CABELlO, P.; CHAMORRO, C. A.; SANDOVAL, J.; FERNADEZ, F. Comparative scanning electron-microscopic study of the lingual papillae in two species of domestic mammals (Equus caballus and Bostaurus). Acta Anatomica, Basel, v. 132, p. 120$123,1988$.

DYCE, K. M., SACK, W. O.; WENSING, C. J. G. Tratado de anatomia veterinária. 2 ed. Rio de Janeiro: Guanabara Koogan, 1997. $813 \mathrm{p}$.

EERDUNCHAOLU, K.; TAKEHANA, K.; YAMAMOTO, E.; KOBAYASHI, A.; CAO, G.; BAIYIN UEDA, H.; TANGKAWATTANA, P. Characteristics of dorsal lingual papillae of the Bactrian camel (Camelus bactrianus). Anatomia, Histologia, Embryologia, Berlin, v. 30, p. 147-151, 2001.

EMURA, S.; HAYAKAWA, D.; CHEN, H.; SHOUMURA, S. Morphology of the lingual papillae in the tiger. Okajimas Folia Anatomica Japonica, Tokyo, v. 81, p. 39-44, 2004.

EMURA, S.; OKUMURA, T.; CHEN, H.; SHOUMURA, S. Morphology of the lingual papillae in the raccoon dog and fox. Okajimas Folia Anatomica Japonica, Tokyo, v. 83, p. 73-76, 2006.

EMURA, S.; OKUMURA, T.; CHEN, H. Morphology of the lingual papillae in the Japanese marten. Okajimas Folia Anatomica Japonica, Tokyo, v. 84, p. 77-82, 2007.

EMURA, S.; OKUMURA, T.; CHEN, H. Morphology of the lingual papillae in the roan antelope. Okajimas Folia Anatomica Japonica, Tokyo, v. 88, n. 3, p. 127-131, 2011a.

EMURA, S.; OKUMURA, T.; CHEN, H. Morphology of the lingual papillae in the Patagonian cavy. Okajimas Folia Anatomica Japonica, Tokyo, v. 88, n. 3, p. 121-125, 2011 b.

GUIMARÃES, G. C.; MACHADO, M. R. F.; SANTOS, A. L. Q. Morfologia, topografia e distribuição das papilas linguais valadas em eqüinos (Equus Caballus, Linnaeus, 1758) sem raça definida. Bioscience Journal, Uberlândia, v. 23, p. 105-110, 2007.

GUIMARÃES, J. P.; MARI, R. B.; MARIGO, J.; ROSAS, F. C. W.; WATANABE, I. Gross and microscopic observations on the lingual structure of the franciscana (Pontoporia blainvillei - Gervais and
d'Orbigny, 1844). Microscopy Research and Technique, New York, v. 75, p. 737-742, 2012.

HARKNERS, J. E.; WAGNER, J. E. Biologia e clinica de coelhos e roedores. 3 ed. São Paulo: Roca, 1993. 238 p.

HERRERA, A. D. P. N.; SANTIAGO, G. S.; MEDEIROS, S. L. S. Importância da fibra na nutrição de coelhos. Ciência Rural, Santa Maria, v. 31, p. 557-561, 2001.

IINO, T.; KOBAYASHI, K. Morphological studies on the lingual papillae and their connective tissue papillae of rat. Shigaku = Odontology, Tokyo, v. 75, p. 1039-1060, 1988.

IWASAKI, S. Evolution of the structure and function of the vertebrate tongue. Journal of Anatomy, London, v. 201, p. 1-13, 2002.

JACKOWIAK, H.; GODYNICKI, S. The scanning electron microscopic study of lingual papillae in the silver fox (Vulpes vulpes fulva, Desmarest, 1820). Annals of Anatomy - Anatomischer Anzeiger, Jena, v. 186, p. 179-183, 2004.

JACKOWIAK, H.; GODYNICKI, S. K.; SKIERESZ-SZEWCZYK, K.; SKA-LORYCH, T. J. Scanning electron microscopic study of the lingual papillae in the arctic fox (Alopex lagopus L., 1758), Anatomia, Histologia, Embryologia, Berlin, v. 38, p. 377-381, 2009.

KEASTER, J. Studies on the anatomy and physiology of the tongue. The Laryngoscope, New York, v. 50, p. 222-258, 1940.

KILINC, M.; ERDOGAN, S.; KETANI, S.; KETANI, M. A. Morphological study by scanning electron microscopy of the lingual papillae in the middle east blind mole rat (Spalax ehrenbergi, Nehring, 1898), Anatomia, Histologia, Embryologia, Berlin, v. 39, p. 509-515, 2010.

KIM, J. Y.; MOCHIZUKI, A. T.; AKITA, B. Q.; JUNGA, H. S. Morphological evidence of the importance of epithelial tissue during mouse tongue development. Experimental Cell Research, Stockholm, v. 290, p. 217-226, 2003.

KOBAYASHI, K. Three-dimensional architecture of the connective tissue core of the lingual papillae in the guinea pig. Anatomia, Histologia, Embryologia, Berlin, v. 182, p. 205-213, 1990.

KOBAYASHI, K.; KUMAKURA, M.; YOSHIMURA, K.; NONAKA, K.; MURAYAMA, T.; HENNEBERG, M. Comparative morphological study of the lingual papillae and their connective tissue cores of the Koala. Anatomia, Histologia, Embryologia, Berlin, v. 206, p. 247-254, 2003.

KOBAYASHI, K.; KUMAKURA, M.; YOSHIMURA, K.; TAKAHASHI, M.; ZHENG, J. H.; KAGEYAMA, I.; KOBAYASHI, K. I.; HAMA, N. Comparative morphological studies on the stereo structure of the lingual papillae of selected primates using scanning electron microscopy. Annals of Anatomy - Anatomischer Anzeiger, Jena, v. 186, p. 525-530, 2004.

KONIG, H. E.; LIEBICH, H. G. Anatomia dos animais domésticos: texto e atlas colorido. Porto Alegre: Artmed, 2004. $787 \mathrm{p}$.

KULAWIK, M.; GODYNICKI, S. Fungiform papillae of the tongue in the rabbit (Oryctolagus cuniculus). Polish Journal Veterinary Sciences, Olsztyn, v. 10, p. 25-27, 2007 a.

KULAWIK, M.; GODYNICKI, S. Vallate papillae in the domestic rabbit (Oryctolagus cuniculus $f$. domestica). Polish Journal Veterinary Sciences, Olsztyn, v. 10, p. 47-50, 2007 b. 
KUMAR, P.; KUMAR S.; SINGH, Y. Tongue papillae in goat: a scanning electron-microscopic study. Anatomia, Histologia, Embryologia, Berlin, v. 27, p. 355-357, 1998.

KUMAR, S.; BATE, L. Scanning electron microscopy of the tongue papillae in the pig (Sus scrofa). Microscopy Research and Technique, New York, v. 63, p. 253-258, 2004.

KURTUL, I.; ATALGIN, S. H. Scanning electron microscopic study on the structure of the lingual papillae of the Saanen goat. Small Ruminant Research, Amsterdam, v. 80, p. 52-56, 2008.

MAHABADY, M. K.; MORAVVATI, H.; KHAZAEIL, K. A microscopic study of lingual papillae in Iranian Buffalo (Bubalus bubalus). Asian Journal of Animal and Veterinary Advances, New York, v. 5, p. 154-161, 2010.

MANÇANARES, C. A. F.; SANTOS, A. C.; PIEMONTE, M. C.; VASCONCELOS, B. G.; CARVALHO, A. F.; MIGLINO, M. A.; AMBRÓSIO, C. E.; ASSIS NETO, A. C. Macroscopic and microscopic analysis of the tongue of the common opossum (Didelphis marsupialis). Microscopy Research and Technique, New York, v. 75, p. 1329-1333, 2012.

NASR, E. S.; GAMAL, A. M.; ELSHEIKH, E. H. Light and scanning electron microscopic study of the dorsal lingual papillae of the rat Arvicanthis niloticus (Muridae, Rodentia), Journal of American Science, New York, v. 8, n. 4, p. 619-627, 2012.

NICKEL, R.; SCHUMMER, A.; SEIFERLE, E. Digestive system. In: SCHUMMER, A., NICKEL, R. (Ed.). The viscera of domestic mammals. 2. ed. Berlim-Hamburg: Verlag Paul Parey, 1979. p. 2126.

NONAKA, K.; ZHENG, J.; KOBAYASHI, K. Comparative morphological study on the lingual papillae and their connective tissue cores in rabbits. Okajimas Folia Anatomica Japonica, Tokyo, v. 85, p. 57-66, 2008.

OJIMA, K. Functional role and angioarchitectural arrangement of the filiform and fungiform papillae on the medial-dorsal surface of the beagle dog tongue. Annals of Anatomy - Anatomischer Anzeiger, Jena, v. 183, p. 325-329, 2001.

OJIMA, K.; MITSUHASHI, F.; NASU, M.; SUZUKI, Y. Functional angioarchitectural comparison of the fungiform papillae of rat, rabbit, cat in scanning electron microscopic specimens. Annals of Anatomy - Anatomischer Anzeiger, Jena, v. 182, p. 451-457, 2000 .
PASTOR, J. F.; BARBOSA, M.; DE PAZ, F. J. Morphological study of the lingual papillae of the giant panda (Ailuropoda melanoleuca) by scanning electron microscopy. Journal of Anatomy, London, v. 212, p. 99-105, 2008.

SHINDO, J.; YOSHIMURA, K.; KOBAYASHI, K. Comparative morphological study on the stereo-structure of the lingual papillae and their connective tissue cores of the American beavers. Okajimas Folia Anatomica Japonica, Tokyo, v. 84, p. 127-138, 2006.

SILVA, M. C. P.; WATANABE, I.; KRONKA, M. C. Threedimensional architecture of the connective tissue core and surface structures of the lingual papillae in the rabbit. Histology and Histopathology, Murcia, v. 17, p. 455-461, 2002.

SISSON, S. Aparelho digestório. In: GETTY, R. (Ed.). Anatomia dos animais domésticos. 5. ed. Rio de Janeiro: Guanabara, 1986. p. 101-107.

SONNTAG, C. F. The comparative anatomy of the tongues of the Mammalia - 1. General description of the tongue. Proceedings of the Zoological Society of London, London, v. 90, p. 115-129, 1920.

SOUZA, A. F.; OLIVEIRA, V. C.; SANTOS A. C.; ROSA, R. A.; CARVALHO, A. F.; AMBRÓSIO, C. E.; MANÇANARES, C. A. F. Morfologia macro e microscópica das papilas linguais do quati, Pesquisa Veterinária Brasileira, Rio de Janeiro, v. 32, n. 3, p. 271-277, 2012.

STANGL, F. B.; PFAU, R. S. Gross morphology and distribution patterns of lingual papillae in some geomyid and heteromyid rodents. Proceedings of the Oklahoma Academy Science, Norman, v. 74, p. 25-29, 1994.

TADJALLI, M.; PAZHOOMAND, R. Tongue papillae in lambs: a scanning electron microscopic study. Small Ruminant Research, Amsterdam, v. 54, p. 157-164, 2004.

ZHENG, J. H.; KOBAYASHI, K. Comparative morphological study on the lingual papillae and their connective tissue cores (CTC) in reeves' muntjac deer (Muntiacus reevesi). Annals of Anatomy Anatomischer Anzeiger, Jena, v. 188, p. 555-564, 2006. 